\title{
Study of 'Fingerprints' for Green Tea from Different Planting Areas in Eastern China
}

\author{
Hai-Yan Liu ${ }^{a} \mathbb{D D}^{\S}$, Lin Ma ${ }^{b}$, Xing-Heng Li $i^{a}$, Jun-Min Fana, Xing-Ping Lv ${ }^{a}$, \\ Zheng $\mathrm{CaO}^{\mathrm{a}}$ and Xue-Song Zhang ${ }^{\mathrm{a}, \mathrm{*}}$ \\ ${ }^{a}$ Tea and Food Science and Technology Institute, Jiangsu Vocational College of Agriculture and Forestry, Jurong 212400, China. \\ ${ }^{b}$ Sericultural Research Institute, College of Biotechnology, Jiangsu University of Science and Technology, Zhenjiang 212018, China. \\ Received 9 May 2019, revised 24 April 2020, accepted 11 November 2020.
}

\begin{abstract}
Green tea is one of the main teas in China, which is unfermented and retains more natural substances of fresh tea leaves. This is the preliminary study of application of 'fingerprints' based on differences in component composition of green tea. Five green teas from different areas in eastern China are analyzed, which are processed by microwave-assisted solvent (ethanol) extraction method to obtain tea polyphenols, flavonoids, polysaccharides, pigments (thearubigins, theaflavins, theabrownins). The results show that the component composition of five green teas are varied from each other; based on these contents varieties, we have constructed a 'fingerprint' and applied linear discriminant analysis (LDA) and hierarchical cluster analysis (HCA) to assist in the identification of these five green teas. This method does not require large, expensive instruments (such as high performance liquid chromatograph, gas chromatograph, mass spectrometer, etc.), and is easy to use, which provides a new avenue for the identification of tea.
\end{abstract}

KEYWORDS

Green tea, contents varieties, identification, 'fingerprints'.

\section{Introduction}

Tea is currently the most popular and healthy non-alcoholic beverage in the world, its consumption is second only to potable water. ${ }^{1}$ Tea contains a variety of natural active ingredients, such as polyphenols, polysaccharides, pigments, flavonoids, alkaloids, proteins, amino acids, etc., ${ }^{2}$ which might be the reason for its antioxidant activity, ${ }^{3}$ antibacterial activity, ${ }^{4}$ anti-obesity, ${ }^{5}$ anti-cancer, ${ }^{6}$ and other biological activities. ${ }^{7}$ Green tea is an unfermented tea with the lowest degree of oxidation and is particularly effective in fighting cancer and cardiovascular diseases. ${ }^{8}$

Green teas from different regions are varied in climate, soil, altitude, harvesting season, etc., thus their contents of active ingredients will change dramatically, which will affect the quality of tea. ${ }^{9}$ The analysis of green tea has recently garnered attention because of its importance in the pharmaceuticals, cosmetics, food additives and functional food. ${ }^{10-11}$ Many methods are available for green tea detection, such as high performance liquid chromatography (HPLC), ${ }^{12}$ gas chromatography (GC), ${ }^{13}$ mass spectrometry (MS), ${ }^{14}$ GC-MS, ${ }^{15}$ gene mapping, ${ }^{16}$ spectroscopic fingerprint, ${ }^{17}$ and so on. ${ }^{18-20}$ All these methods have high selectivity and great sensitivity for tea detection. However, these methods often require more complex sample pretreatment, environmentally unfriendly chemicals, expensive equipment, professional technicians, which limits their application in the simple and rapid analysis of green tea.

Here, we report a novel 'fingerprint' based on the variety of bioactive ingredients to discriminate green teas from different regions, which can provide different patterns to realize the selectivity. Green teas from five different regions are processed by microwave-assisted solvent extraction, and then the extracts are evaluated to obtain the contents of bioactive ingredients as

*To whom correspondence should be addressed. E-mail: shelz@sina.com polyphenols, flavonoids, pigments (thearubigins, theaflavins, theabrownins) and polysaccharides. In addition, linear discriminant analysis (LDA) and hierarchical cluster analysis (HCA) are applied for comprehensive consideration of interference from various factors to improve the applicability and specificity of the method. The method described herein has several advantages as follows: (1) it is simple and cost-effective, which requires no skilled operators and sophisticated instruments; (2) the determination can be achieved easily by extracting bioactive ingredients from different green teas, which are reusable to discover their nutritional values; (3) it is safe and green, no need of environmentally unfriendly chemicals. Based on these advantages, our method may provide a new way for the detection of biological samples.

\section{Materials and Methods}

\subsection{Reagents and Instruments}

All the reagents used were of analytical-reagent grade. Water was deionized and further purified with a thermo scientific water purification system (Lab Tower EDI 15, Sweden). Green teas of five different regions (high-grade green tea of Wuxi in Jiangsu province, Mingqian maojian of Mount Wuyi in Fujian province, Mingyuan spring buds of Jurong in Jiangsu province, Biluochun of Dongting Hill in Jiangsu province, Jinzhai Cuimei of Ta-pieh Mountains in Anhui province) were bought at a supermarket in Jurong, China. Absolute ethanol and sodium nitrite were obtained from Tianjin Kermel Chemical Reagent Co., Ltd. (Tianjin, China). Rutin hydrate, epigallocatechin gallate (EGCG), gallocatechin (EC), catechin (C), aluminum nitrate nonahydrate and gallic acid were from Macklin Biochemical Co., Ltd. (Shanghai, China). Sodium hydroxide, hydrogen peroxide $30 \%$, ethyl acetate, folinol reagent, sodium carbonate, sodium bicarbonate, oxalic acid, phenol, sulfuric acid were from 
XiLong Chemical Co., Ltd. (Shantou, China). The epigallocatechin (EGC) and epicatechin gallate (ECG) were from Aladdin Reagent Co., Ltd. (Shanghai, China). The methyl alcohol was from TEDIA company Inc. (Fairfield, USA). The acetonitrile was from Merck KGaA (Darmstadt, Germany)

The UV-vis absorption data were recorded using a UV-visible spectrophotometer (755B, Shanghai Precision Instrument Co., Ltd., China). The centrifugation was conducted by a high-speed centrifuge (TG 16-WS, Shanghai Lu Xiangyi Centrifuge Instrument Co., Ltd., China). The microwave process was completed with a multifunctional microwave chemical reactor (MWave5000, Shanghai Sineo Microwave Chemistry Technology Co., Ltd., China). The identification and quantification of polyphenols was achieved by a high performance liquid chromatograph (ACQUITY Arc, Waters Technology Co., Ltd., USA). The evaporation and concentration were done by a rotary evaporator (RE-5203, Shanghai Yarong Biochemistry Instrument Factory, China).

\subsection{Preparation of Tea Extractions}

One gram of each green tea powder was processed with microwave-assisted ethanol solvent extraction, respectively. Under specific conditions (microwave chemical reactor power $400 \mathrm{~W}$, liquid-solid ratio $35 \mathrm{~mL} \mathrm{~g}^{-1}$, temperature $65^{\circ} \mathrm{C}$ and time $3 \mathrm{~min}$ ), the bioactive ingredients were extracted; after the extraction process, the solutions were centrifuged at $4000 \mathrm{rpm}$ for $10 \mathrm{~min}$; the precipitates were processed for a second time under the same conditions; finally the two supernatants were collected in a reagent bottle, concentrated and vacuum-dried to obtain the extracts.

\subsection{Content Determination of Active Ingredients}

The content determination of polyphenols, total flavonoids, pigments, polysaccharides in extracts were carried out referring to Folin-Ciocalteu colorimetry, ${ }^{21}$ aluminum nitrate complex spectrophotometry, ${ }^{22}$ Roberts assay, ${ }^{23}$ phenol-sulfuric acid assay. ${ }^{24}$ All the raw data of standard curve for the quantification are shown in the Supplementary Material (Fig. S1, S2 and S3). Each experiment was conducted in five replicates.

\subsection{Identification and Quantification of Polyphenols with High Performance Liquid Chromatography (HPLC)}

Chromatographic conditions: The separation was achieved using a CORTECS-C18 column $(2.7 \mu \mathrm{m}, 100 \times 3.0 \mathrm{~mm})$ with a UV-visible light detector at wavelength of $280 \mathrm{~nm}$. The mobile phase for gradient elution consisted of a acetonitrile-water system, wherein mobile phase A is ultrapure water, and mobile phase $\mathrm{B}$ is acetonitrile. The conditions of the gradient elution were 0-3.0 $\mathrm{min}, 9.0 \% \mathrm{~B}$; 3.0-6.0 $\mathrm{min}, 9.0-20 \% \mathrm{~B}$; 6.0-8.0 $\mathrm{min}$ 20.0-30.0 \% B; 8.0-9.0 min, 30.0-9.0\% B; 9.0-20.0 min, $9.0 \%$ B. The volume of injection was $10 \mu \mathrm{L}$, the column temperature was $30{ }^{\circ} \mathrm{C}$, and the system delivered a constant flow of $300 \mu \mathrm{L} \mathrm{min}^{-1}$.

\section{Results and Discussion}

3.1. 'Fingerprints' of Five Green Teas from Different Regions According to the method in Section 2.3, the bioactive ingredients' contents of five green teas (high-grade green tea, Mingqian maojian, Mingyuan spring buds, Biluochun, and Jinzhai Cuimei) were obtained. The raw data are shown in Table S1. In Fig. 1, the differently coloured squares represent different bioactive ingredients' contents (using mean values, $\mathrm{n}=5$ ), and a column of coloured squares constitute a 'fingerprint' map for each green tea, which is similar to the DNA fingerprints in

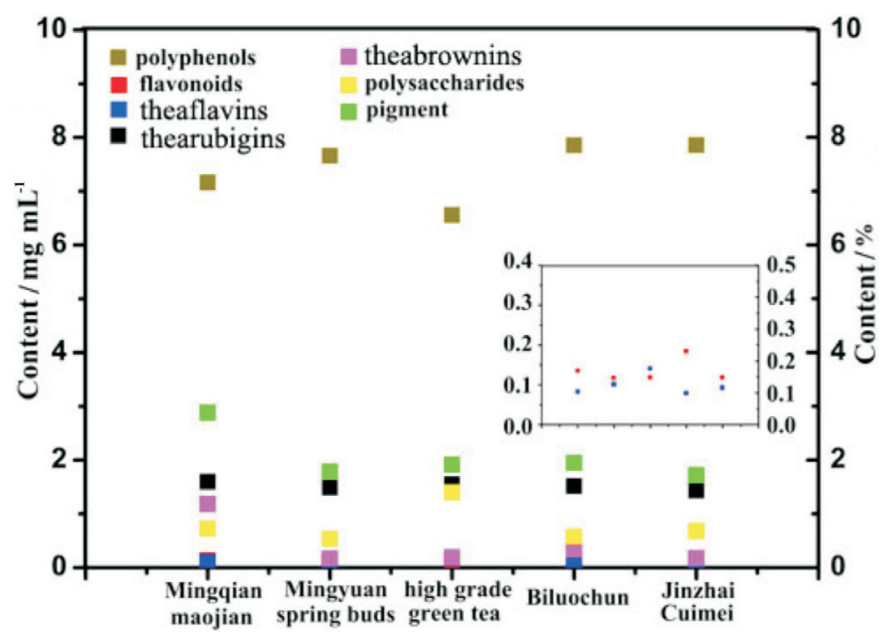

Figure 1 'Fingerprints' of five target green teas (using mean values, $\mathrm{n}=$ 5); the insert shows the partial enlargement.

molecular biology. It can also be seen clearly that the five green teas contain a lot of polyphenols; there are more theabrownins than polysaccharides only in Mingqian maojian; the content of theabrowins, pigments and thearubigins is almost equal in highgrade green tea, and Jinzhai Cuimei and Mingyuan spring buds have the similar contents of pigments and thearubigins. Above all, the 'fingerprints' of five green teas are different and could be used for the identification.

\subsection{Linear Discriminant Analysis (LDA)}

In order to make the tea discrimination more simple, the initial data of the 'fingerprints' (Fig. 1) were processed by LDA, according to a multi-dimensional matrix ( 5 green teas $\times 7$ ingredients $\times$ 5 replicates). As a classical linear discrimination method, LDA can transform sample data into a three-dimensional or twodimensional version displayed as a scatter plot. ${ }^{25}$ In the plot, data of the same specimen gather around a centre to form a cluster (Fig. 2); clusters of various specimens stay far from each other. Through this method, the separation of the specimens can be enhanced. From Fig. 2, it can be seen that each tea has its own spherical-cluster and distant from others (spheres represent sample data generated from 'fingerprints' of teas). The qualitative information on the teas can be achieved based on the position of the clusters presented in the plot. Five green teas are

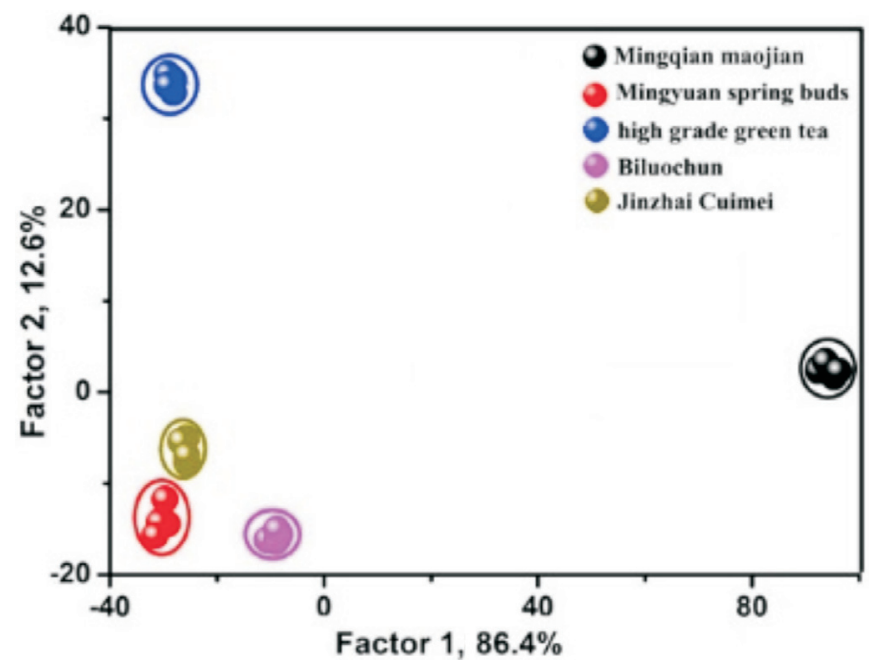

Figure 2 Canonical score plot for the five teas, which is provided by LDA according to a multi-dimensional matrix ( 5 green teas $\times 7$ ingredients $\times$ 5 replicates). 
successfully differentiated in the LDA, with a classification accuracy of $100 \%$ according to a leave-one-out classification matrix (Table S2 and S3, Supplementary Material).

\subsection{Hierarchical Cluster Analysis (HCA)}

In addition, we have applied HCA to further explore the intrinsic similarities between different green teas. HCA can deeply explore the intrinsic relationship between data, and gradually aggregate the samples according to the similarity of their qualities. The ones with the highest similarity are preferentially aggregated together, showing the inter-class spacing (Euclidean distance) smaller; the least similar is then aggregated together, showing a larger inter-class spacing. ${ }^{26}$ As shown in Fig. 3, the samples are obviously separated into two groups: A and B; Jinzhai Cuimei and Biluochun have a minimum euclidean distance less than 5, followed by Mingyuan spring buds and high-grade green tea as the euclidean distance close to 10 . The reason might be that Jinzhai Cuimei and Biluochun are both

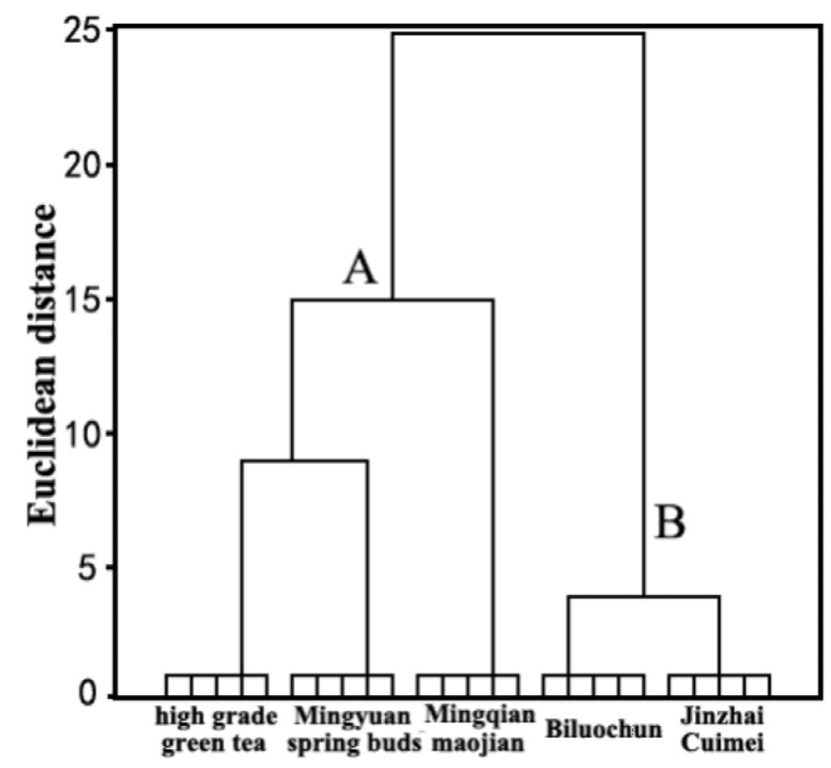

Figure 3 Hierarchical cluster analysis of five target green teas, according to a multi-dimensional matrix ( 5 green teas $\times 7$ ingredients $\times 5$ replicates). from mountainous areas and affected by a similar topography, temperature and humidity; high-grade green tea and Mingyuan spring buds are from Wuxi and Jurong, respectively, which are hilly terrain and affected by similar climatic conditions; with the influence of various comprehensive factors, such as climate, topography, soil conditions, etc., it eventually leads to other fareuclidean distances.

\subsection{HPLC Analysis}

Recently, more and more studies show that green tea contains a variety of bioactive ingredients, including polyphenols, flavonoids, pigments, polysaccharides, etc. ${ }^{2,27}$ Kodama's group have found that catechins are one of the major tea polyphenols, which account for 20-30\% of the dry weight, such as (-)-epigallocatechin gallate (EGCG), (-)-epigallocatechin (EGC), (-)-epicatechin gallate (ECG), (-)-gallocatechin (EC) and $(+)$-catechin (C). ${ }^{27}$ Herein, HPLC analysis was carried out to determine the composition of polyphenols in green tea. The results are shown in Table 1, indicating that high-grade green tea contains a large amount of $C$, and a certain amount of ECG, EGC and EGCG; Mingyuan spring buds consisted of C, EGCG and ECG; there are four kinds of polyphenols in Biluochun and Mingqian maojian, such as C, EGCG, ECG and EGC; there are a lot of C/EGCG and a small amount of ECG in Jinzhai cuimei. These results are in consistent with former ones obtained by Folin-Ciocalteu colorimetry to a certain degree. Each experiment was conducted in triplicate. Certainly, there are some other polyphenols, which will be studied in our later research. All the raw data are shown in Fig. S4-9 in the Supplementary Material. The specific components and other characterizations of our findings need further research in the future. More studies on other samples will also be done in future.

\section{Conclusion}

In the present study, the discrimination of five green teas from different regions based on 'fingerprints' map was established based on the contents of active ingredients [polyphenols, flavonoids, polysaccharides, pigments (thearubigins, theaflavins, theabrownins)] in green teas, which is green, simple and cost-effective; in addition, linear discriminant analysis (LDA) and hierarchical cluster analysis (HCA) were applied to discrimi-

Table 1 Contents of polyphenols $\left(\mathrm{mg} \mathrm{mL}^{-1}\right)$ in the green teas.

\begin{tabular}{|c|c|c|c|c|c|c|c|}
\hline Samples & Component & $\begin{array}{l}\text { Retention } \\
\text { time }\end{array}$ & $\begin{array}{l}\text { Content } \\
/ \mathrm{mg} \mathrm{mL}^{-1}\end{array}$ & Samples & Component & $\begin{array}{l}\text { Retention } \\
\text { time }\end{array}$ & $\begin{array}{l}\text { Content } \\
/ \mathrm{mg} \mathrm{mL}^{-1}\end{array}$ \\
\hline \multirow[t]{5}{*}{ High grade green tea } & EGC & 3.85 & $0.26 \pm 0.04$ & \multirow[t]{5}{*}{ Mingqian maojian } & EGC & 3.80 & $0.29 \pm 0.05$ \\
\hline & C & 4.78 & $3.80 \pm 0.21$ & & C & 4.73 & $3.08 \pm 0.26$ \\
\hline & EGCG & 9.38 & $1.29 \pm 0.03$ & & EGCG & 9.30 & $1.47 \pm 0.15$ \\
\hline & ECG & 11.51 & $0.44 \pm 0.03$ & & ECG & 11.48 & $0.45 \pm 0.06$ \\
\hline & Total & - & 5.79 & & Total & - & 5.29 \\
\hline \multirow[t]{4}{*}{ Jinzhai cuimei } & $\mathrm{C}$ & 4.76 & $3.68 \pm 0.23$ & \multirow[t]{4}{*}{ Mingyuan spring buds } & C & 4.69 & $4.58 \pm 0.22$ \\
\hline & EGCG & 9.32 & $3.06 \pm 0.25$ & & EGCG & 9.25 & $1.42 \pm 0.14$ \\
\hline & ECG & 11.49 & $0.50 \pm 0.02$ & & ECG & 11.46 & $0.55 \pm 0.03$ \\
\hline & Total & - & 7.24 & & Total & - & 6.55 \\
\hline \multirow[t]{5}{*}{ Biluochun } & EGC & 3.76 & $0.31 \pm 0.03$ & & & & \\
\hline & C & 4.70 & $4.51 \pm 0.28$ & & & & \\
\hline & ECG & 11.47 & $0.61 \pm 0.06$ & & & & \\
\hline & EGCG & 9.25 & $2.07 \pm 0.18$ & & & & \\
\hline & Total & - & 7.50 & & & & \\
\hline
\end{tabular}

Note: the retention time of standards are as follows (EGC: $3.85 \mathrm{~min}, \mathrm{C}: 4.70 \mathrm{~min}, \mathrm{EC}: 8.79 \mathrm{~min}, \mathrm{EGCG}: 9.30 \mathrm{~min}, \mathrm{ECG}: 11.48 \mathrm{~min}$ ); if the retention time of a component in the sample solution differs from that of a standard solution within $\pm 0.1 \mathrm{~min}$, the component can be identified as the standard. 
nate green teas more intuitively, which laid a foundation for the later analysis of more 'fingerprints' mapping of tea in China.

\section{Acknowledgements}

The authors gratefully acknowledge the financial supports by Jiangsu Province Higher Education College Students Innovation and Entrepreneurship Training Program (201913103004Y and 201713103002Y).

\section{Supplementary Material}

Supplementary information is provided in the online supplement.

\section{${ }^{8}$ ORCID ID}

H.-Y. Liu:

orcid.org/0000-0002-4963-793X

\section{Reference}

1 P.L. Fernández-Cáceres, M.J. Martín and F. Pablos, et al., Differentiation of tea (Camellia sinensis) varieties and their geographical origin according to their metal content, J. Agric. Food Chem., 2001, 49, 4775-4779.

2 A. Crocker, Tea, Ullmann's Encyclopedia of Industrial Chemistry, 2000, $35,679-684$.

3 N. Naumovski, B.L. Blades and P.D. Roach, Food inhibits the ora bioavailability of the major green tea antioxidant epigallocatechin gallate in humans, Antioxidants, 2015, 4, 373-393.

4 R. Díaz-Gomez, H. Toledo-Araya, R. Lopez-Solís, et al., Combined effect of gallic acid and catechin against Escherichia coli, LWT-Food Sci. Technol., 2014, 59, 896-900.

5 Y.H. Kao, H.H. Chang, M.J. Lee, et al., Tea, obesity, and diabetes, Mol. Nutr. Food Res., 2006, 50, 188-210.

6 Y.H. Cao and R.H. Cao, Angiogenesis inhibited by drinking tea, Nature, 1999, 398, 381.

7 T.O. Cheng, All teas are not created equal the Chinese green tea and cardiovascular health, Int. J. Cardiol., 2006, 108, 301-308.

8 V. Stangl, M. Lorenz and K. Stangl, The role of tea and tea flavonoids in cardiovascular health, Mol. Nutr. Food Res., 2006, 50, 218-228.

9 J. Lee, D.H. Chambers and E. Chambers IV, A comparison of the flavor of green teas from around the world, J. Sci. Food. Agr., 2014, 94, 1315-1324.

10 B.J. An, J.H. Kwak, J.H. Son, et al., Biological and anti-microbial activity of irradiated green tea polyphenols, Food Chem., 2004, 88, 549-555.

11 S.P. Huang and S.D. Huang, Dynamic hollow fiber protected liquid phase microextraction and quantification using gas chromatography combined with electron capture detection of organochlorine pesticides in green tea leaves and ready-to-drink tea, J. Chromatogr. A, 2006, 1135, 6-11.

12 K.B. Wang, F. Liu, Z.H. Liu, et al., Comparison of catechins and volatile compounds among different types of tea using high performance liquid chromatograph and gas chromatograph mass spectrometer, 2011, 46, 1406-1412.
13 G. Ntlhokwe, A. G.J. Tredoux, T. Górecki, et al., Analysis of honeybush tea (Cyclopia spp.) volatiles by comprehensive two-dimensional gas chromatography using a single-stage thermal modulator, Anal. Bioanal. Chem., 2017, 409, 4127-4138.

14 K. Fraser, S.J. Harrison, G.A. Lane, et al., Analysis of low molecular weight metabolites in tea using mass spectrometry-based analytical methods, Crit. Rev. Food Sci., 2014, 54, 924-937.

15 W. Pongsuwan, E. Fukusaki, T. Bamba, et al., Prediction of Japanese green tea ranking by gas chromatography/mass spectrometry-based hydrophilic metabolite fingerprinting, J. Agric. Food Chem., 2007, 55, 231-236.

16 C.L. Wei, H. Yang, S.B. Wang, et al., Draft genome sequence of Camellia sinensis var. sinensis provides insights into the evolution of the tea genome and tea quality, Proc. Natl. Acad. Sci., 2018, 115, 41514158.

17 G.G. Buyukgoz, M. Soforoglu, N.B. Akgul and I.H. Boyaci, Spectroscopic fingerprint of tea varieties by surface enhanced Raman spectroscopy, J. Food Sci. Technol., 2016, 53, 1709-1716.

18 W.D. Dai, D.D. Qi, T. Yang, et al., Non-targeted analysis using ultra-performance liquid chromatography quadrupole-time of flight mass spectrometry uncovers the effects of harvest season on the metabolites and taste quality in tea (Camellia sinensis L.), J. Agric. Food Chem., 2015, 63(44), 9869-9878.

19 G. Martínez-Domínguez, R. Romero-González and A.G. Frenich, Multi-class methodology to determine pesticides and mycotoxins in green tea and royal jelly supplements by liquid chromatography coupled to Orbitrap high resolution mass spectrometry, Food Chem., 2016, 197, 907-915.

20 N. Seetohul, S.M. Scott, W.T O'Hare, et al., Discrimination of Sri Lankan black teas using fluorescence spectroscopy and linear discriminant analysis, J. Sci. Food Agric., 2013, 93, 2308-2314.

21 E.A. Ainsworth and K.M. Gillespie, Estimation of total phenolic content and other oxidation substrates in plant tissues using Folin-Ciocalteu reagent, Nature Protoc., 2007, 2, 875-877.

22 T.H. Kao and B.H. Chen, Functional components in soybean cake and their effects on antioxidant activity, J. Agri. Food Chem., 2006, 54, 7544-7555.

23 E.A.H. Roberts and R.F. Smith, Spectrophotometric measurements of theaf lavins and thearubigins in black tea liquors in assessments of quality in teas, Analyst, 1961, 1019, 94-98.

24 M. Dubois, K.A. Gilles, J.K. Hamilton, et al., Colorimetric method for determination of sugars and related substances, Anal.Chem., 1956, 28 , 350-356.

25 H.Y. Liu, L. Ma, S.H. Xu, W.H. Hua and J. Ouyang, Using metal nanoparticles as a visual sensor for the discrimination of proteins, J. Mater. Chem. B, 2014, 2, 3531-3537.

26 W. Revelle, Hierarchical cluster analysis and the internal structure of tests, Multivar. Behav. Res., 1979, 14, 57-74.

27 D.H. Kodama, A.E.S.S. Gonçalves, F.M. Lajolo and M.I. Genovese, Flavonoids, total phenolics and antioxidant capacity: comparison between commercial green tea preparations, Food Sci. Technol., 2010, 30, 1077-1082. 


\section{Supplementary material to:}

H.-Y. Liu, L. Ma, X.-H. Li, J.-M. Fan, X.-P. Lv, Z. Cao and X.-S. Zhang,

Study of 'Fingerprints' for Green Tea from Different Planting Areas in Eastern China, S. Afr. J. Chem., 2021, 75, 28-31. 


\section{Supplementary Material}

For

\section{Study on "Fingerprints" for Green Tea from Different Planting Areas in Eastern China}

Hai-Yan Liu', Lin Ma ${ }^{\mathrm{b}}$, Xing-Heng Li ${ }^{\mathrm{a}}$, Jun-Min Fan ${ }^{\mathrm{a}}$, Xing-Ping Lv ${ }^{\mathrm{a}}$, Zheng Cao ${ }^{\mathrm{a}}$, Xue-Song Zhang ${ }^{\mathrm{a} *}$

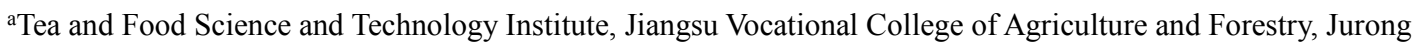
212400, China.

bSericultural Research Institute, College of Biotechnology, Jiangsu University of Science and Technology, Zhenjiang 212018, China.

\section{Materials and methods}

\section{The standard curve for determination of polyphenols}

The tea extracts' polyphenols were determined by using Folin-Ciocalteu colourimetry and using gallic acid as the standard. The gallic acid solution was diluted into a range of different concentrations, respectively mixed with some colour-forming reagents, and then the absorbance was measured at $765 \mathrm{~nm}$. Three parallel tests were done at each concentration. The standard curve was prepared by plotting the concentration against the absorbance, shown in Figure S1.

The results showed that the standard curve equation is $\mathrm{y}=0.0165 \mathrm{x}+0.0102$ (where $\mathrm{x}$ is the sample concentration, $\mathrm{y}$ is the mean absorbance of sample, $\mathrm{r}=0.9980$ ). 


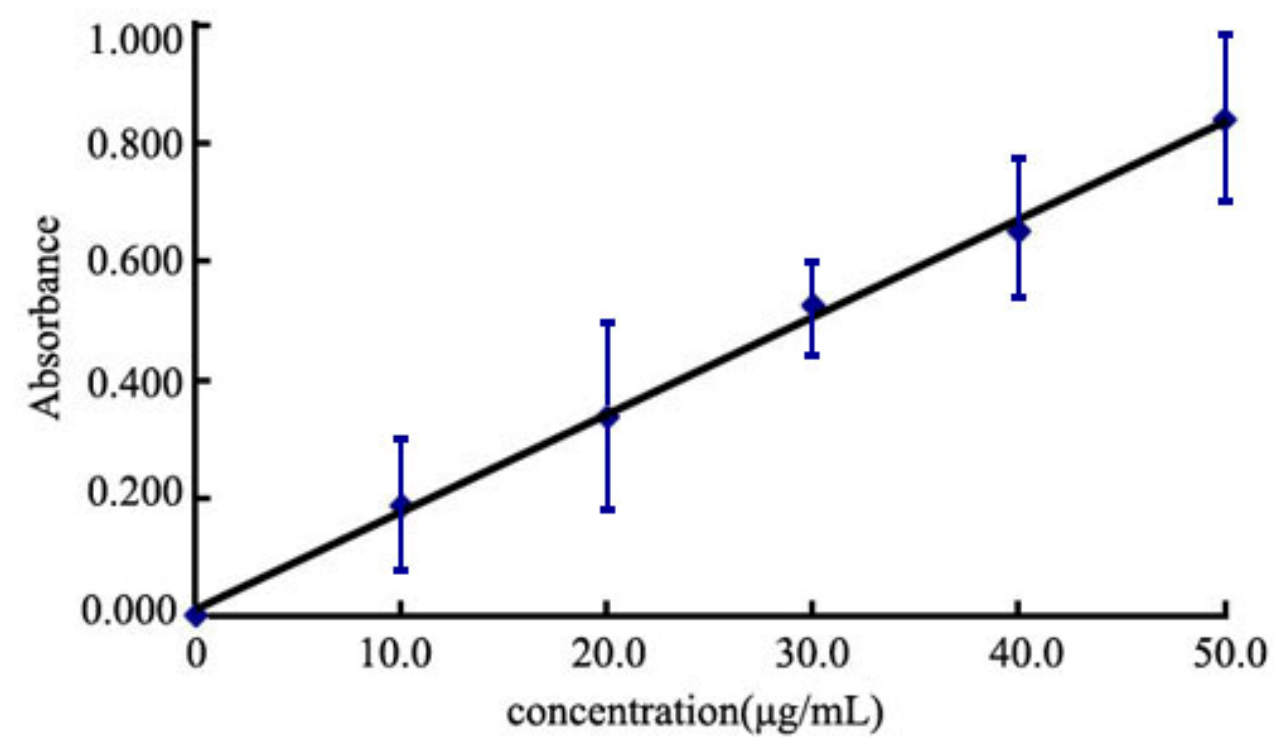

Figure S1. The standard curve of gallic acid, values are means \pm RSD $(\%), n=3$ tests at gallic acid concentrations of $0,10.0,20.0,30.0,40.0$, and $50.0 \mu \mathrm{g} / \mathrm{mL}$.

\section{The standard curve for determination of total flavonoids}

To quantify the flavonoids extracted from tea, we carried out the experiments to obtain the rutin standard curve. The rutin standard solution was diluted into a range of different concentrations, respectively mixed with some colour-forming reagents, and then measured the absorbance at $510 \mathrm{~nm}$. By plotting concentration against absorbance, the standard curve was prepared, which is shown in Figure S2. It could be seen that the linear dynamic range of rutin was from 0 to $0.05 \mathrm{mg} / \mathrm{mL}$, and the equation of the standard curve is $\mathrm{y}=9.07 \mathrm{x}-0.004$ (where $\mathrm{x}$ is the sample concentration, $\mathrm{y}$ is the absorbance of sample, $r=0.9987$ ). All the data were taken in triplicate, the means and relative standard deviation (\%) values were calculated. 


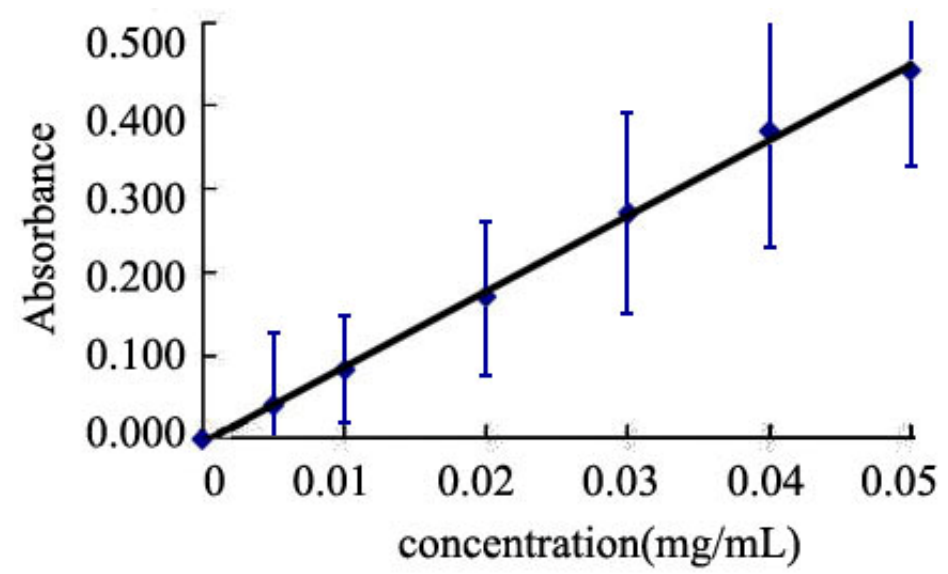

Figure S2. The standard curve of rutin, values are means $\pm \operatorname{RSD}(\%), \mathrm{n}=3$ tests at rutin.

concentrations of $0,0.005,0.01,0.02,0.03,0.04$, and $0.05 \mathrm{mg} / \mathrm{mL}$.

\section{The determination of pigments}

The determination of pigments, such as total pigments, theaflavins (TFs), theabrownins (TBs), thearubigins (TRs), was referred to the Roberts assay with slight modification. $0.2 \mathrm{~g}$ of tea extract was diluted into $100 \mathrm{~mL}$ with water for preparing solutions A, B, C and D. Solution A: first pipette $25 \mathrm{ml}$ of the former diluent, add $25 \mathrm{~mL}$ of ethyl acetate, then shake and stand for a few minutes to get $2 \mathrm{~mL}$ of ethyl acetate extract, lastly add $23 \mathrm{~mL}$ of $95 \%$ ethanol to the ethyl acetate extract. Solution C: get the former ethyl acetate extract of $15 \mathrm{~mL}$, then add $15 \mathrm{~mL}$ of $2.5 \% \mathrm{NaHCO}_{3}$ solution, then shake and stand for a few minutes to get $4 \mathrm{~mL}$ of ethyl acetate extract, at last add $21 \mathrm{~mL}$ of $95 \%$ ethanol to the ethyl acetate extract. Solution B: take $2 \mathrm{~mL}$ of the former aqueous solution (from the process of making solution A), add $2 \mathrm{~mL}$ of the saturated oxalic acid solution and $6 \mathrm{~mL}$ of water, lastly add $15 \mathrm{~mL}$ of $95 \%$ ethanol to the solution. Solution D: pipette $25 \mathrm{~mL}$ of the former diluent, add $25 \mathrm{~mL}$ of $\mathrm{n}$-butanol, then shake and let it stand for a few minutes to form a $2 \mathrm{~mL}$ water layer, add $2 \mathrm{~mL}$ of the saturated oxalic acid solution and $6 \mathrm{~mL}$ of water, lastly add $15 \mathrm{~mL}$ of $95 \%$ ethanol to the solution. Each solution's absorbance was measured at a wavelength of $380 \mathrm{~nm}$ by using $95 \%$ ethanol as a blank. 
The formulas for the determination of pigments concentrations were as follows:

TFs $(\%)=2.25 \times \mathrm{Ac} \times 100 \% / \mathrm{m}$

TRs $(\%)=7.06 \times(2 \mathrm{Aa}+2 \mathrm{Ad}-\mathrm{Ac}-2 \mathrm{Ab}) \times 100 \% / \mathrm{m}$

TBs $(\%)=2 \times \operatorname{Ad} \times 7.06 \times 100 \% / m$

Here, $\mathrm{m}$ was the sample weight; Aa, Ab, Ac, Ad was the absorbance of solution A, B, C and D, respectively; 2.25 and 7.06 were the conversion factors under the same operating conditions.

\section{The standard curve for determination of polysaccharides}

The tea extracts' polysaccharides were determined by the phenol sulfuric acid assay method using glucose as the standard. The glucose solution was diluted into a range of different concentrations by mixing with some colour-forming reagents, and then the absorbance was measured at $490 \mathrm{~nm}$. Three parallel tests were done at each concentration. By plotting the concentration against absorbance, the standard curve shown in Figure S3 was prepared.

The results showed that the standard curve equation is $\mathrm{y}=1.3618 \mathrm{x}-0.0197$ (where $\mathrm{x}$ is the sample concentration, $\mathrm{y}$ is the mean absorbance of sample, $\mathrm{r}=0.9966$ ).

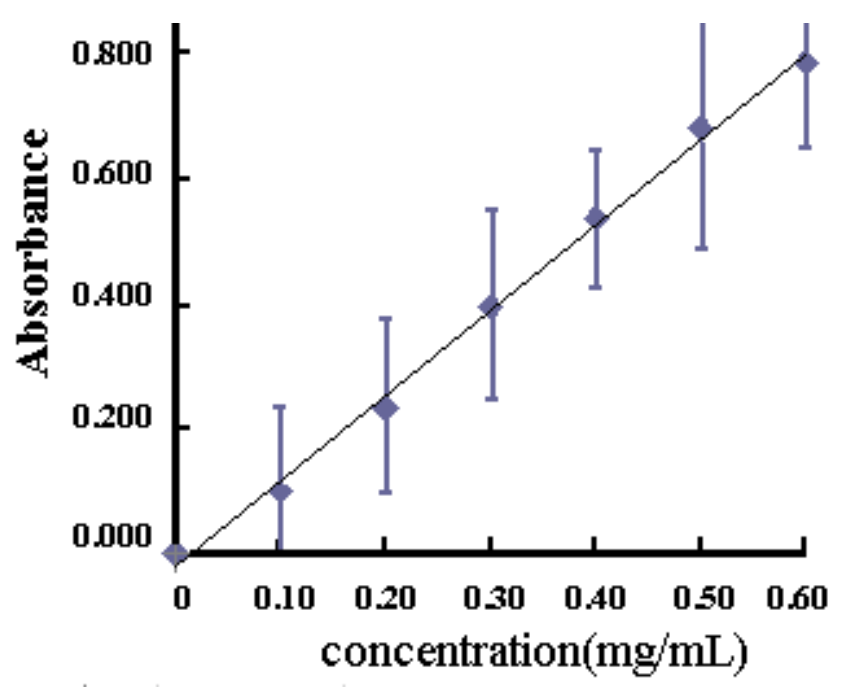

Figure S3. The standard curve of glucose, values are means \pm RSD $(\%), n=3$ tests at gallic acid concentrations of $0,0.10,0.20,0.30,0.40,0.50$ and $0.60 \mathrm{mg} / \mathrm{mL}$. 
5. The raw data of the green tea active ingredients

Table S1. The contents of the green tea active ingredients.

\begin{tabular}{|c|c|c|c|c|c|c|c|}
\hline & $\begin{array}{c}\text { flavonoids } \\
\mathrm{mg} / \mathrm{mL} \\
\end{array}$ & $\begin{array}{c}\text { polyphenols } \\
\mathrm{mg} / \mathrm{mL}\end{array}$ & $\begin{array}{c}\text { theaflavins } \\
\% \\
\end{array}$ & $\begin{array}{c}\text { theabrownins } \\
\% \\
\end{array}$ & $\begin{array}{c}\text { thearubigins } \\
\% \\
\end{array}$ & $\begin{array}{c}\text { pigment } \\
\% \\
\end{array}$ & $\begin{array}{c}\text { polysaccharides } \\
\% \\
\end{array}$ \\
\hline \multirow{5}{*}{$\begin{array}{l}\text { Mingqian } \\
\text { maojian }\end{array}$} & 0.1378 & 7.21 & 0.108 & 1.165 & 1.566 & 2.839 & 0.715 \\
\hline & 0.1347 & 7.11 & 0.104 & 1.201 & 1.624 & 2.929 & 0.718 \\
\hline & 0.1358 & 7.13 & 0.1 & 1.168 & 1.61 & 2.878 & 0.733 \\
\hline & 0.1362 & 7.15 & 0.107 & 1.176 & 1.578 & 2.861 & 0.728 \\
\hline & 0.135 & 7.19 & 0.102 & 1.198 & 1.597 & 2.897 & 0.723 \\
\hline \multirow{5}{*}{$\begin{array}{c}\text { Mingyuan } \\
\text { spring } \\
\text { buds }\end{array}$} & 0.1072 & 7.58 & 0.118 & 0.154 & 1.49 & 1.742 & 0.519 \\
\hline & 0.1162 & 7.62 & 0.123 & 0.172 & 1.48 & 1.775 & 0.515 \\
\hline & 0.1264 & 7.63 & 0.134 & 0.174 & 1.491 & 1.799 & 0.528 \\
\hline & 0.1198 & 7.77 & 0.129 & 0.168 & 1.501 & 1.798 & 0.523 \\
\hline & 0.1203 & 7.68 & 0.132 & 0.176 & 1.483 & 1.791 & 0.578 \\
\hline \multirow{5}{*}{$\begin{array}{c}\text { high } \\
\text { grade } \\
\text { green tea }\end{array}$} & 0.1285 & 6.6 & 0.177 & 0.194 & 1.539 & 1.91 & 1.382 \\
\hline & 0.1187 & 6.54 & 0.169 & 0.184 & 1.542 & 1.895 & 1.405 \\
\hline & 0.1092 & 6.54 & 0.176 & 0.186 & 1.536 & 1.898 & 1.392 \\
\hline & 0.1199 & 6.43 & 0.179 & 0.189 & 1.562 & 1.93 & 1.412 \\
\hline & 0.1204 & 6.67 & 0.182 & 0.193 & 1.553 & 1.928 & 1.409 \\
\hline \multirow{5}{*}{ Biluochun } & 0.1632 & 7.87 & 0.093 & 0.325 & 1.513 & 1.931 & 0.553 \\
\hline & 0.2034 & 7.85 & 0.102 & 0.343 & 1.517 & 1.962 & 0.588 \\
\hline & 0.1836 & 7.83 & 0.09 & 0.307 & 1.503 & 1.9 & 0.563 \\
\hline & 0.179 & 7.92 & 0.099 & 0.336 & 1.52 & 1.955 & 0.572 \\
\hline & 0.192 & 7.79 & 0.112 & 0.351 & 1.532 & 1.995 & 0.588 \\
\hline \multirow{5}{*}{$\begin{array}{l}\text { Jinzhai } \\
\text { Cuimei }\end{array}$} & 0.1354 & 7.83 & 0.117 & 0.169 & 1.404 & 1.69 & 0.643 \\
\hline & 0.1043 & 7.89 & 0.114 & 0.171 & 1.423 & 1.708 & 0.643 \\
\hline & 0.1038 & 7.83 & 0.111 & 0.167 & 1.436 & 1.714 & 0.703 \\
\hline & 0.1225 & 7.79 & 0.121 & 0.175 & 1.454 & 1.75 & 0.711 \\
\hline & 0.1305 & 7.93 & 0.119 & 0.182 & 1.442 & 1.743 & 0.693 \\
\hline
\end{tabular}


6. The linear discriminant analysis (LDA) and cross-validation with leave-one-out classification matrix

Table S2. Training matrix obtained from the sensor array against 5 kinds of green tea samples. LDA was carried out and resulting in factors of the canonical scores and group generation. Crossvalidation with leave-one-out classification showed the $100 \%$ correct classification

\begin{tabular}{cccc}
\hline $\begin{array}{c}\text { Analytes } \\
\text { Green tea }\end{array}$ & $\begin{array}{c}\text { Results LDA (The first two factors) } \\
\text { Factor } \mathbf{1}\end{array}$ & Factor $\mathbf{2}$ & Group \\
\hline Mingqian maojian & 92.47 & 2.68 & 1 \\
Mingqian maojian & 95.28 & 1.71 & 1 \\
Mingqian maojian & 92.32 & 2.36 & 1 \\
Mingqian maojian & 93.66 & 3.38 & 1 \\
Mingqian maojian & 96.11 & 2.32 & 1 \\
Mingyuan spring buds & -30.34 & -14.08 & 2 \\
Mingyuan spring buds & -29.25 & -14.42 & 2 \\
Mingyuan spring buds & -30.80 & -14.28 & 2 \\
Mingyuan spring buds & -31.54 & -15.72 & 2 \\
Mingyuan spring buds & -29.70 & -11.73 & 2 \\
high grade green tea & -28.12 & 32.86 & 3 \\
high grade green tea & -28.15 & 34.26 & 3 \\
high grade green tea & -28.47 & 34.38 & 3 \\
high grade green tea & -29.29 & 34.85 & 3 \\
high grade green tea & -29.25 & 33.63 & 3 \\
Biluochun & -9.03 & -16.33 & 4 \\
Biluochun & -8.25 & -15.61 & 4 \\
Biluochun & -10.58 & -16.18 & 4 \\
Biluochun & -8.82 & -16.25 & 4 \\
Biluochun & -8.81 & -15.04 & 5 \\
Jinzhai Cuimei & -25.69 & -7.69 & 5 \\
Jinzhai Cuimei & -25.59 & -7.76 & 5 \\
Jinzhai Cuimei & -25.75 & -5.06 & 5 \\
Jinzhai Cuimei & -26.76 & -5.35 & 5 \\
Jinzhai Cuimei & -25.65 & -6.93 & 5 \\
\hline
\end{tabular}


Table S3. The results of leave-one-out classification matrix ${ }^{\text {a,c }}$

\begin{tabular}{|c|c|c|c|c|c|c|c|c|}
\hline & & \multicolumn{6}{|c|}{ Predicted group membership } & \multirow{2}{*}{ Total } \\
\hline & & Group & 1 & 2 & 3 & 4 & 5 & \\
\hline \multirow[t]{10}{*}{ Original } & Number & 1 & 5 & 0 & 0 & 0 & 0 & 5 \\
\hline & & 2 & 0 & 5 & 0 & 0 & 0 & 5 \\
\hline & & 3 & 0 & 0 & 5 & 0 & 0 & 5 \\
\hline & & 4 & 0 & 0 & 0 & 5 & 0 & 5 \\
\hline & & 5 & 0 & 0 & 0 & 0 & 5 & 5 \\
\hline & $\%$ & 1 & 100.0 & 0 & 0 & 0 & 0 & 100.0 \\
\hline & & 2 & 0 & 100.0 & 0 & 0 & 0 & 100.0 \\
\hline & & 3 & 0 & 0 & 100.0 & 0 & 0 & 100.0 \\
\hline & & 4 & 0 & 0 & 0 & 100.0 & 0 & 100.0 \\
\hline & & 5 & 0 & 0 & 0 & 0 & 100.0 & 100.0 \\
\hline Cross & Number & 1 & 5 & 0 & 0 & 0 & 0 & 5 \\
\hline \multirow[t]{9}{*}{ Validation $^{\mathrm{b}}$} & & 2 & 0 & 5 & 0 & 0 & 0 & 5 \\
\hline & & 3 & 0 & 0 & 5 & 0 & 0 & 5 \\
\hline & & 4 & 0 & 0 & 0 & 5 & 0 & 5 \\
\hline & & 5 & 0 & 0 & 0 & 0 & 5 & 5 \\
\hline & $\%$ & 1 & 100.0 & 0 & 0 & 0 & 0 & 100.0 \\
\hline & & 2 & 0 & 100.0 & 0 & 0 & 0 & 100.0 \\
\hline & & 3 & 0 & 0 & 100.0 & 0 & 0 & 100.0 \\
\hline & & 4 & 0 & 0 & 0 & 100.0 & 0 & 100.0 \\
\hline & & 5 & 0 & 0 & 0 & 0 & 100.0 & 100.0 \\
\hline
\end{tabular}

a: $100 \%$ of the original grouping observations have been correctly classified;

b: Only those observations in the analysis are cross-validated. In Cross-validation, each observation is classified according to a function derived from all observations other than that observation; c: $100 \%$ cross-validation of grouped observations classified correctly. 


\section{HPLC}

\subsection{The standard curve for determination of different polyphenols}

The determination of different polyphenols in tea extracts was carried out by HPLC using an external standard method, taken EGC, C, EC, EGCG and ECG as examples. The standard solutions were diluted into a range of different concentrations $(40-100 \mu \mathrm{g} / \mathrm{mL})$, respectively. Three parallel tests were done at each concentration. The standard curves were prepared by plotting concentration against peak area, which are shown in Figure S4. The results show that the equation of EGC, C, EC, EGCG and ECG is $y=-73760.97+6611.08 x(r=0.99783), y=368298.98+11183.11 x(r=0.99253)$, $y=52217.22+24658.56 x \quad(r=0.99311), \quad y=-312168.93+26823.54 x \quad(r=0.99168), \quad y=-$ 1.14728E6+71669.01x $(\mathrm{r}=0.99573)$ (where $\mathrm{x}$ is the sample concentration, $\mathrm{y}$ is the mean peak area of sample), respectively. 

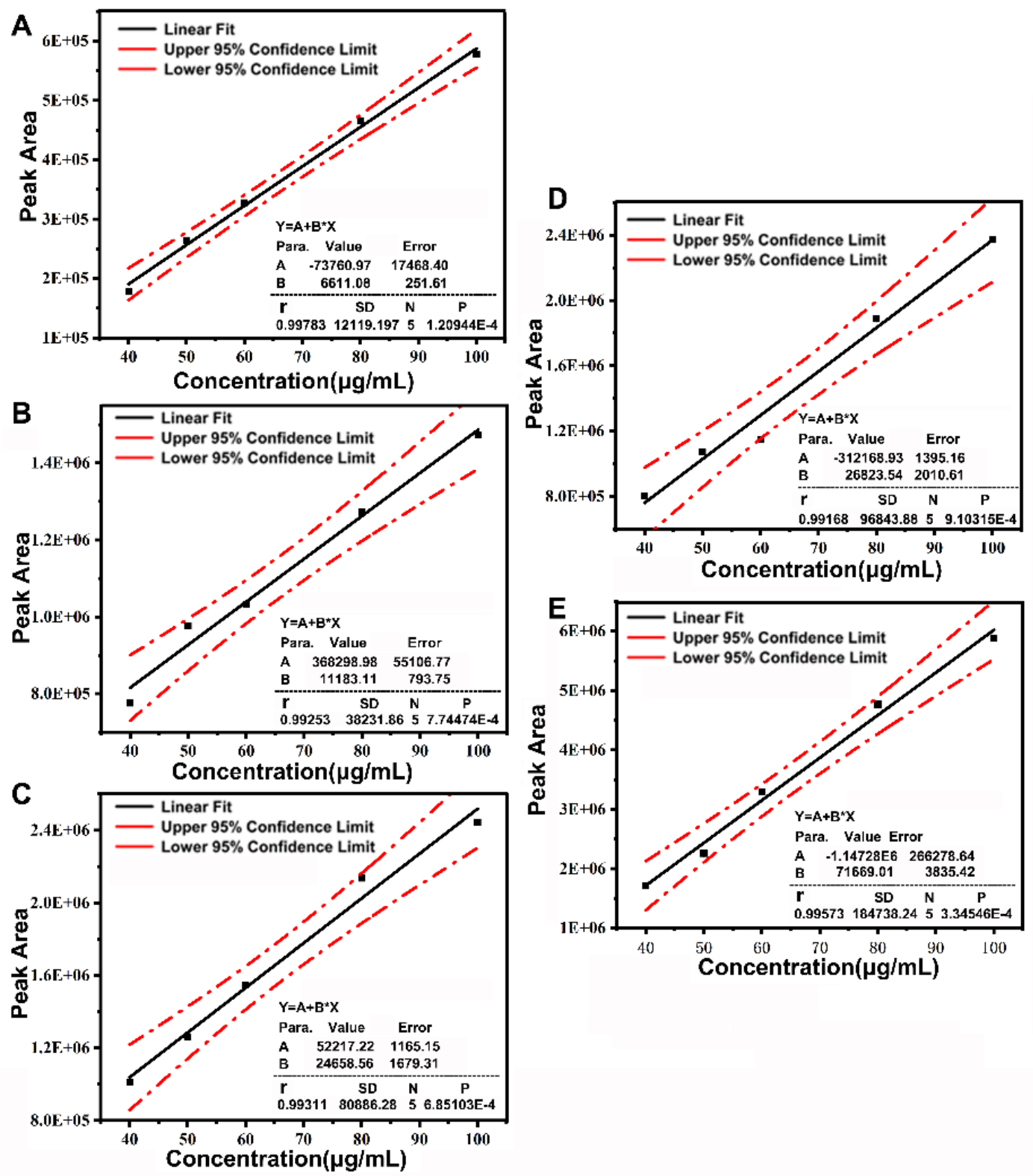

Figure S4. The standard curve of different polyphenols, values are means \pm RSD (\%), $n=3$ tests at concentrations of 40, 50, 60, 80 and $100 \mu \mathrm{g} / \mathrm{mL}$. A: EGC; B: C; C: EC; D: EGCG; E: ECG. 
7.2 The HPLC spectra of different teas

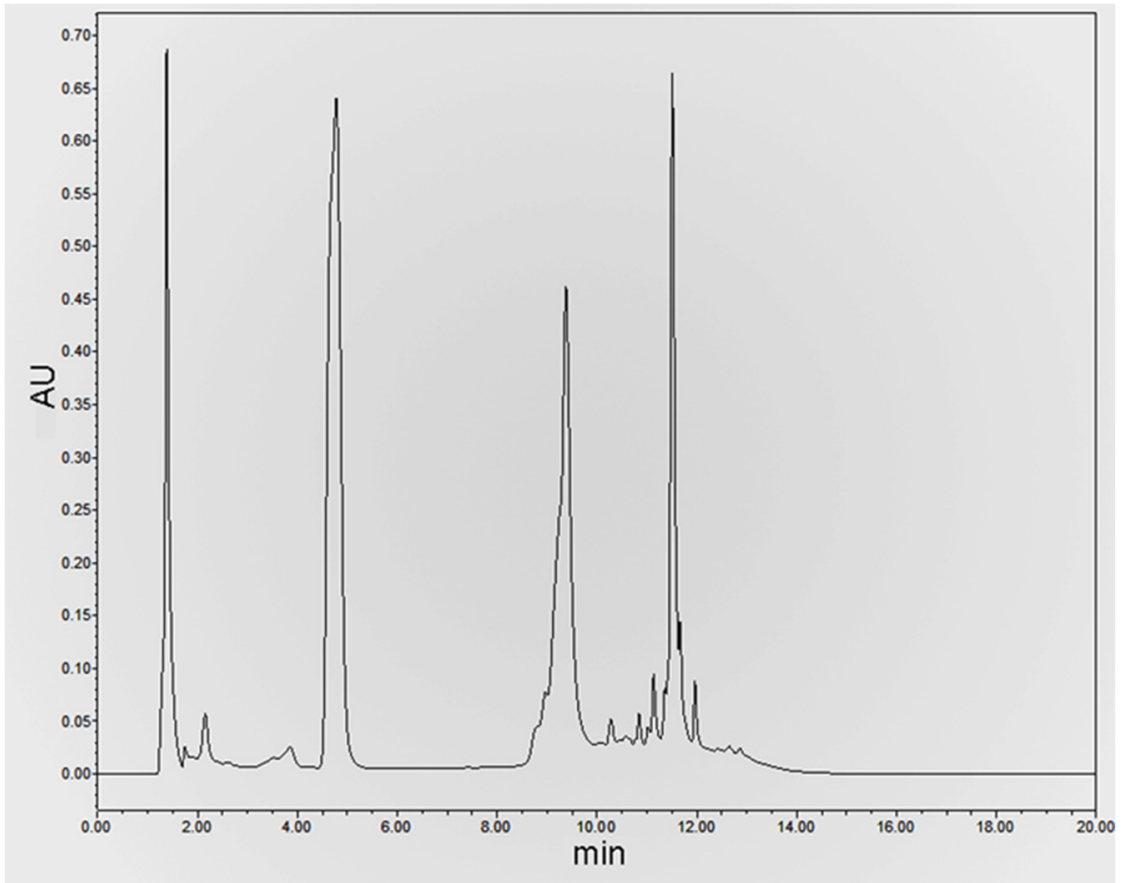

Figure S5. The HPLC spectrum of high-grade green tea.

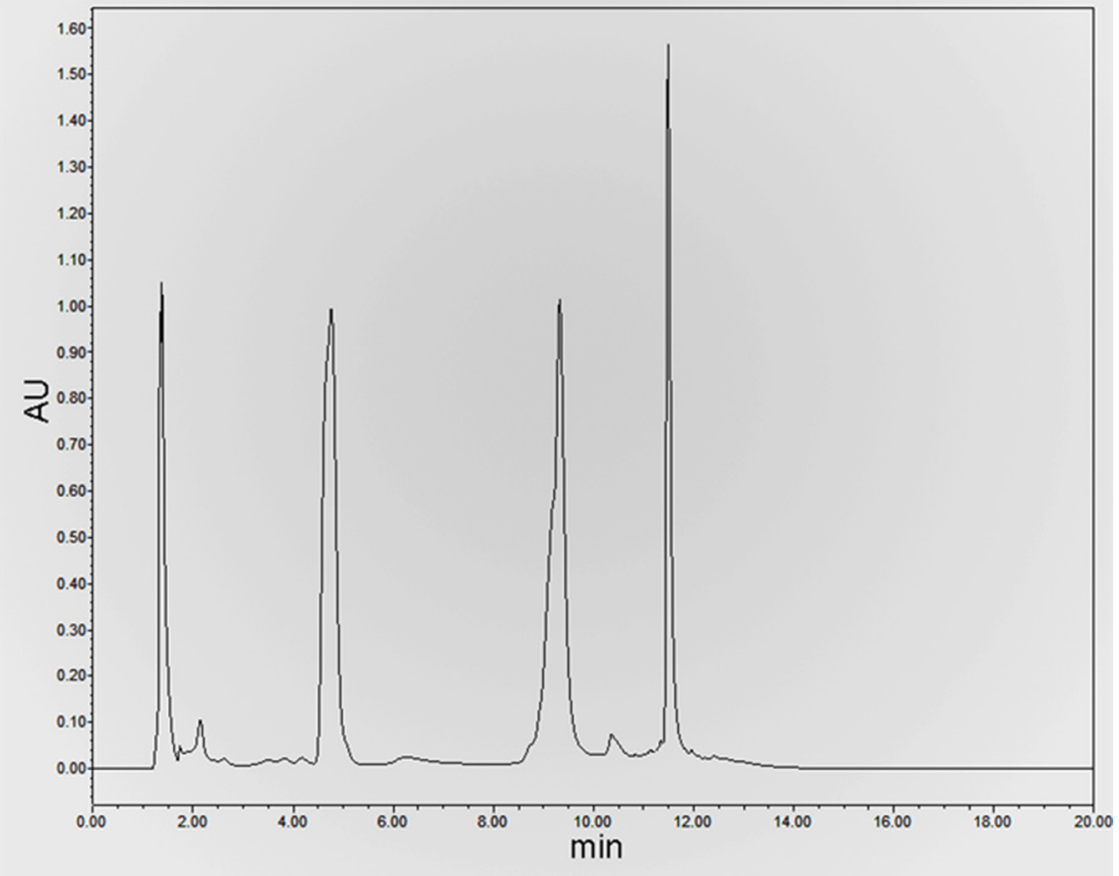

Figure S6. The HPLC spectrum of Jinzhai cuimei. 


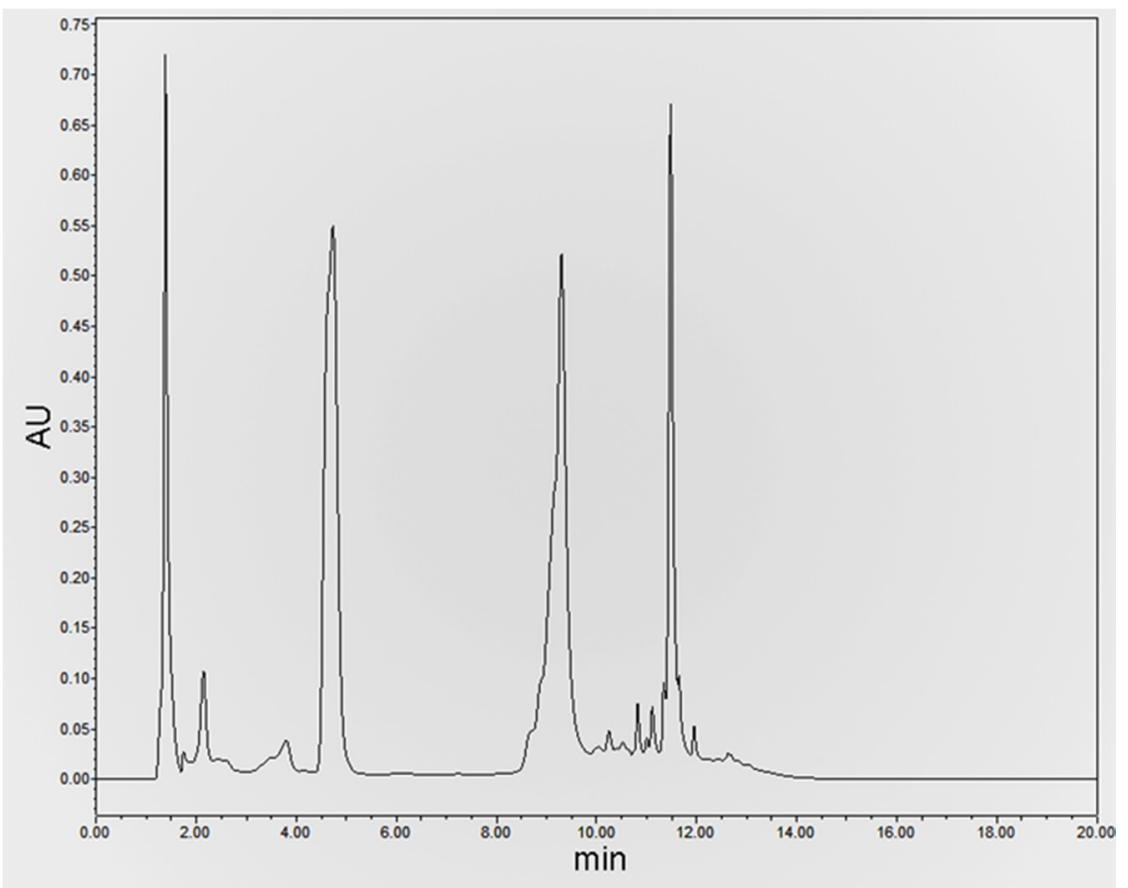

Figure S7. The HPLC spectrum of Mingqian maojian.

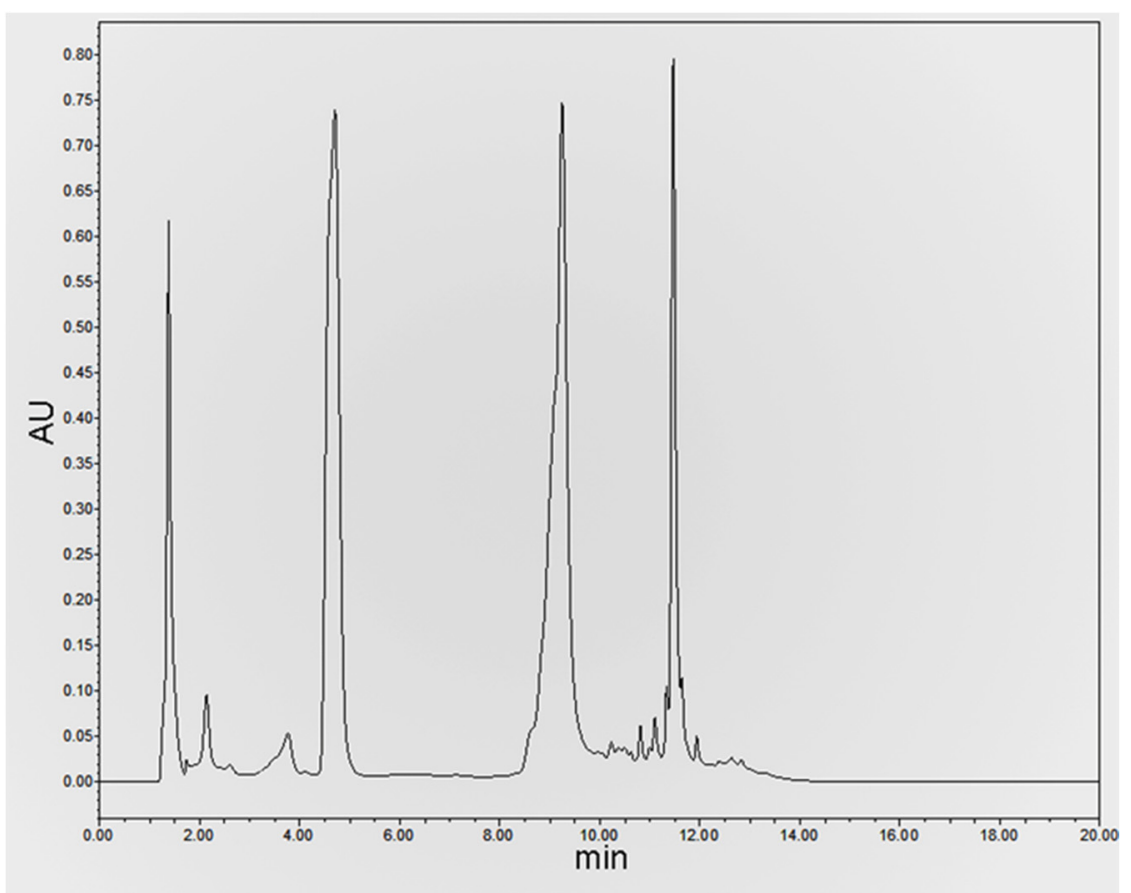

Figure S8. The HPLC spectrum of Biluochun. 


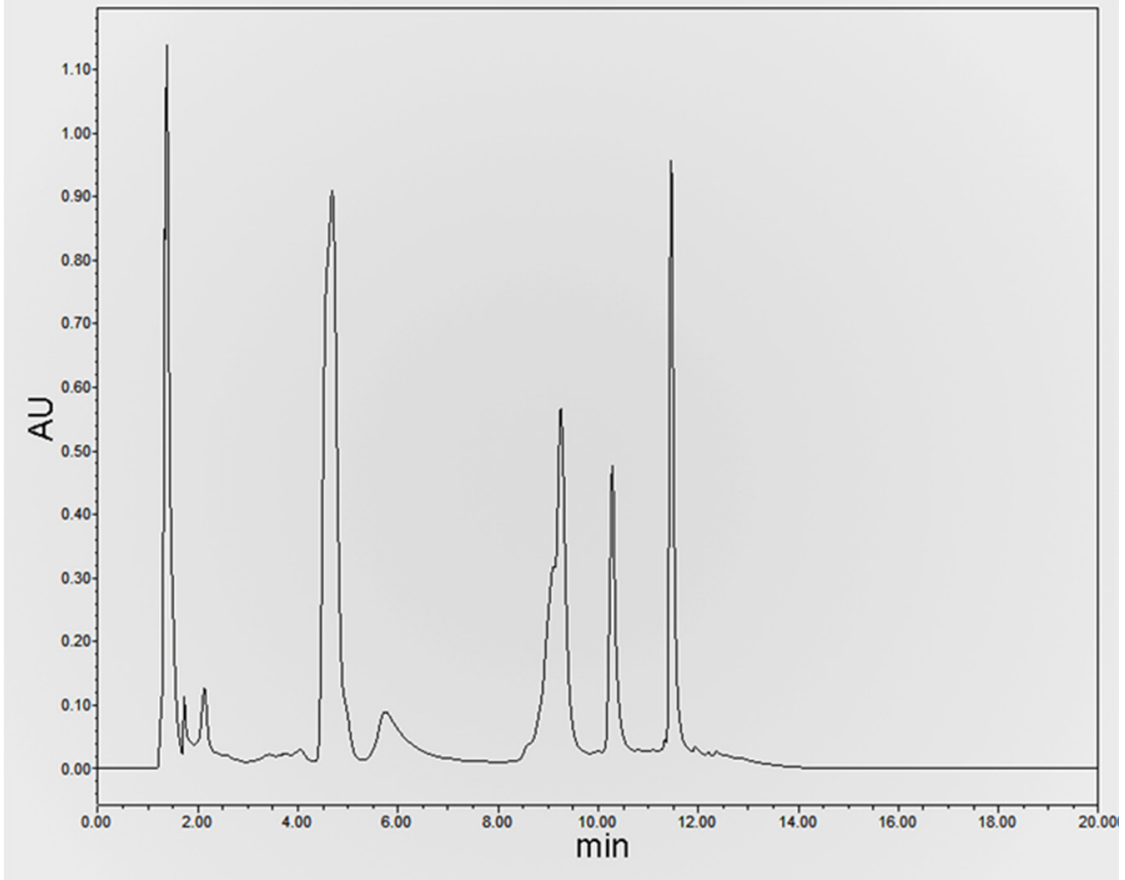

Figure S9. The HPLC spectrum of Mingyuan spring buds. 